\title{
Comunidades de aprendizaje en red: diseño de un proyecto de entorno colaborativo.
}

\author{
Antonia Lozano Díaz \\ alozanodeuoc.edu \\ Universidad de Almería
}

\section{Introducción.}

El movimiento de Comunidades de Aprendizaje surge como reactivo social ante la incapacidad de la educación formal para responder a las nuevas y emergentes necesidades de las generaciones actuales y venideras. Desde este planteamiento se somete a revisión los objetivos, contenidos, y metodologías educativas actuales y, de modo especial, a los agentes educativos, escenarios y fines educativos (Coll, 2004). Aunque bajo el epígrafe Comunidades de Aprendizaje coexisten gran variedad de significados, prácticas y propuestas, pueden detectarse una serie de elementos de relevancia que afectan a alguno de los niveles de la educación formal: las aulas, los centros educativos, el contexto social y comunitario y el uso de las nuevas tecnologías de la información y la comunicación (Coll, ib.).

En la era de la información la forma específica de estructura social es la sociedad de redes. Lo característico de esta nueva sociedad no es el papel de la información y el conocimiento, sino el conjunto de nuevas tecnologías que han permitido a las redes constituirse como "seres" evolutivos con capacidad de adaptación. Lo importante es que las redes, por la estructura que les es inherente, descentralizan la actuación y permiten compartir el proceso de toma de decisiones (Castells, 2001). Desde esta perspectiva, las Comunidades de Aprendizaje pueden incrementar su eficacia si aprovechan la posibilidad que el espacio electrónico ofrece para generar nuevos escenarios educativos y canales de intercambio y participación entre los diferentes implicados.

\section{Internet como escenario educativo.}

La incorporación de las tecnologías de la información y la comunicación a la escuela ha dejado de ser una moda o un lujo para pasar a convertirse en una necesidad para una sociedad potentemente afectada por procesos tecnológicos como la nuestra. Por otro lado, la incorporación educativa del espacio electrónico exige su consideración como el "tercer entorno" de interacción personal y educativa que es (Echeverría, 2002) junto a los otros dos entornos, el rural y el urbano. Diseñar cualquier tipo de proyecto en red, material didáctico multimedia o entorno virtual de aprendizaje precisa, en primer lugar, de un marco teórico que dote de coherencia a las diversas acciones planificadas. El espacio-tiempo virtual tiene sus propias posibilidades y limitaciones que deben ser abordadas convenientemente desde nuestro modelo de enseñaza y de aprendizaje.

\subsection{Comunidades de Aprendizaje en la red.}

Las Comunidades de Aprendizaje parten de un principio regulador, el aprendizaje dialógico. Esta concepción comunicativa defiende que el aprendizaje depende principalmente de las interacciones entre personas, de la construcción conjunta de significados. Un modo de desarrollar el diálogo reflexivo es a través de grupos interactivos. En estos grupos se estimula el cambio de roles, unas veces se enseña y otras se aprende del otro, la cooperación y la colaboración. Esta consideración del papel del alumno se aleja de los enfoques constructivistas de corte más cognitivo, básicamente centrados en los procesos del alumno, y se centra en otros de índole más social. La atención se centra ahora en la dinámica interactiva de co-construcción del conocimiento entre profesor y alumno. 
Desde la perspectiva sociocultural de Vygostky, el ordenador se convierte en una novedosa herramienta para la interacción con la información, el conocimiento y las personas, y el trabajo en grupo uno de las formas ideales para trabajar en la Zona de Desarrollo Próximo (Gross, 2002). Sin embargo el trabajo en grupo de los alumnos requiere de una serie de condiciones para que se produzcan aprendizajes en la dirección deseada entre las que podemos destacar (Durán y Miquel, 2003):

- planificación detallada y compleja del proceso de aprendizaje e interacción grupal.

- Interdepedencia positiva del grupo, el éxito individual está ligado al del grupo y viceversa.

- Uso de las diferencias existentes entre los alumnos como un recurso pedagógico más.

- Consideración de las aportaciones del alumnado como otro elemento básico de calidad junto al profesor.

Una vez establecidas estas premisas psicopedagógicas pasamos a presentar un proyecto de aprendizaje colaborativo en la red, para el desarrollo de estrategias de aprendizaje en materia de lectura. Posteriormente el proyecto se esbozará conforme al modelo metodológico de diseño virtual propuesto por Jochems, Martens y Strijbos (2004).

\section{Justificación del proyecto.}

El movimiento de Comunidades de Aprendizaje presta especial atención al aprendizaje de la lectura como herramienta fundamental de aprendizajes posteriores. Por otro lado, estudios a gran escala como el Proyecto PISA (en su primera edición) en el que se evalúa el rendimiento alumnos/as de 15 años de más de 30 países, entre ellos España, muestran las carencias en comprensión lectora de los adolescentes. En PISA también se consideran el tipo de estrategias de aprendizaje utilizadas por los alumnos destacando de modo importante en el informe que "el aspecto más próximamente relacionado con el rendimiento es el control del proceso de aprendizaje". Como conclusión inmediata se puede observar, por un lado, la necesidad de ayudar al alumno en la adquisición del control de su propio proceso de aprendizaje, y por otro la necesidad de enseñar cómo aplicar este control a actividades como la lectura.

\section{Planteamiento y modelo teórico.}

La metodología instruccional del conocimiento estratégico viene determinado por aspectos clave como los siguientes:

- La enseñanza de las estrategias debe seguir el paradigma constructivista centrado en el aprendizaje más que en la enseñanza, en la construcción de significados más que en la adquisición de contenidos sin más.

- Las características del conocimiento estratégico como contenido de estudio en sí alude a contenidos de carácter procedimental frente a los de carácter declarativo.

- Reestructuración de los papeles del profesor y el alumno. El alumno debe participar activamente en el desarrollo de su conocimiento de un modo autorregulado y autónomo, el profesor debe crear las condiciones instruccionales necesarias para facilitarlo.

El modelo teórico para el entrenamiento instruccional es el Modelo estratégico de Ellis (cfr. Beltrán, 1998). Este autor ha diseñado una estrategia ejecutiva, o meta-estrategia, para facilitar un uso más eficiente de los procesos de aprendizaje significativo en lugar de aprender estrategias diferentes para cada materia y habilidad intelectual. En el corazón de la estrategia está el desarrollar hábitos mentales positivos: dominar la impulsividad, promover la reflexión, organizar y activar el conocimiento previo y abordar las tareas de un modo efectivo y eficiente. Los pasos de la metaestrategia están diseñados para hacer más concretos los procesos cognitivos clave del estudiante poco experto. En síntesis el modelo se basaría en los siguientes pasos de la regulación del aprendizaje: 
1- Preparación para el aprendizaje: pensar antes

- estrategias de planificación.

2- Procesamiento: pensar durante.

- estrategias de supervisión.

3- Consolidación y ampliación del procesamiento: pensar después.

- estrategias de evaluación y revisión.

\section{Aspectos metodológicos para diseñar un entorno colaborativo de trabajo y aprendizaje a} través de Internet.

\subsection{Marco metodológico.}

El trabajo con el alumnado en entornos virtuales alude a principios cosntructivistas como: la resolución de problemas, el trabajo conjunto, el análisis desde diferentes perspectivas, la responsabilidad del propio proceso de aprendizaje y la toma de conciencia del propio papel en el proceso de enseñanza-aprendizaje. Sin embargo no están tan definidas las orientaciones metodológicas sobre cómo diseñar convenientemente un material virtual para el trabajo y aprendizaje en grupo. Jochems, Martens y Strijbos (2004) proponen un marco metodológico para desarrollar entornos CSGBL (Computer-Supported Group-Based Learning), las orientaciones básicas para el diseño son, en síntesis, las siguientes:

- Es preciso centrarse más en el proceso de interacción y aprendizaje que en los resultados.

- Partir de la interacción como elemento clave exige tener en cuenta los elementos críticos que afectan a la misma.

- Los elementos críticos para diseñar entornos virtuales de trabajo y aprendizaje en grupo son:

a. Los objetivos de aprendizaje: el desarrollo de aprendizajes sencillos exigen menos interacción que otros aprendizajes de carácter más complejo (que pueden exigir el uso de estrategias como la argumentación o la discusión).

b. El tipo de tarea: las tareas muy estructuradas (generalmente con una sola solución válida) exigen menos interacción que las poco estructuradas.

c. El grado de predefinición de la interacción: si está demasiado preestructurada la interacción puede resultar artificial o forzada, una escasa estructuración puede hacer que la interacción no sea considerada como un elemento fundamental para el aprendiezaje sino un aspecto meramente anecdótica u opcional.

d. El tamaño del grupo: lo adecuado de su tamaño dependerá de cómo se organice la comunicación, de cómo se diseñe la tarea y de las herramientas disponibles.

e. Las posibilidades tecnológicas: no todo lo tecnológicamente posible tiene porque ser educativamente deseable.

* Explicitar previamente las características de la interacción y diseñar el resto en concordancia.

El proceso de diseño metodológico se orienta a través de seis pasos:

1. determinación de los objetivos de aprendizaje. 
2. determinación de la interacción que se espera.

3. selección del tipo de tarea considerando los objetivos de aprendizaje y la interacción esperada.

4. determinación del grado de preestructuración preciso considerando los objetivos de aprendizaje, la interacción esperada y el tipo de tarea.

5. determinación del tamaño de agrupamiento más apropiado considerando los objetivos de aprendizaje, la interacción esperada, el tipo de tarea y el grado de preestructuración de la interacción.

6. determinación de las posibilidades tecnológicas para apoyar el aprendizaje y la interacción.

Aplicación al desarrollo de una propuesta concreta.

En relación a nuestro proyecto de creación de una comunidad de aprendizaje colaborativo en la red para el desarrollo de estrategias de lectura, los pasos quedarían como sigue:

1. Determinación del tipo de objetivos de aprendizaje:

* Todos los alumnos tienen que trabajar las mismas estrategias, aunque el logro de los objetivos sea diferente.

* Es preciso mostrar un determinado nivel de dominio en el objetivo previo para alcanzar el siguiente, los objetivos están graduados y son interdependientes.

* Las destrezas de trabajo son variadas y abarcan tanto procedimientos complejos (argumentación, negociación, discusión de diferentes alternativas, análisis, planificación, transferencia, síntesis) como otros más sencillos (p.e.aprendizaje de conceptos).

2. Determinación de la interacción:

* Interacción centrada en el intercambio y creación de ideas.

* Interacción entre alumnos y entre profesor y alumnos para recibir feedback del proceso.

* Interacción entre alumnos para la coordinación de actividades de resolución de problemas, de análisis, etc.

3. Selección del tipo de tareas en función de los objetivos de aprendizaje y la interacción propuesta.

* Las tareas para trabajar las destrezas más sencillas serán principalmente actividades estructuradas como la enumeración de ejemplos, aplicación de un procedimiento sencillo, actividades con una única solución válida. Las tareas para trabajar destrezas más complejas implican actividades más ambiguas y menos estructuradas como el estudio de casos, análisis y comentarios, debates, discusión y confrontación de ideas y perspectivas, etc.

* Todos los alumnos deben estudiar el mismo material en relación al tipo de estrategia de aprendizaje que se está estudiando y aplicando, no obstante se pone a su disposición lecturas y materiales adicionales que pueden consultar voluntariamente.

* El tipo de tareas se coordina con los objetivos de aprendizaje para su logro, podrán sufrir modificaciones en función de las necesidades y/o dificultades que vayan surgiendo.

4. Determinación del grado de preestructuración de la interacción considerando los objetivos de aprendizaje, la interacción esperada y el tipo de tarea. 
* Para el logro de objetivos y destrezas más sencillas la interacción entre los sujetos no se prescribe en modo alguno aunque se facilita, el profesor prescribe únicamente la interacción con el contenido.

* Para el logro de las estrategias y destrezas más complejas se prescribe la formación de grupos aunque no el nivel de interacción en ellos, el alumno es libre de elegir el grupo al que quiere pertenecer.

* A lo largo del curso se trabajará preferentemente en grupos, aunque también se hará de modo individual y en gran grupo.

* No se prescriben las funciones de cada uno de los miembros dentro de los grupos, ellos deciden su propia organización y modo de trabajo.

* Las actividades más estructuradas se trabajan de modo individual o mediante interacciones espontáneas, las actividades más ambiguas y menos estructuradas son más apropiadas para ser trabajadas en procesos interactivos predefinidos.

5. Determinación del tamaño de agrupamiento más apropiado considerando los objetivos de aprendizaje, la interacción esperada, el tipo de tarea y el grado de preestructuración de la interacción.

* En las actividades en pequeño grupo (3-5 miembros) se evalúa el rendimiento del grupo. En las actividades en gran grupo (p.e. para la lluvia de ideas) se evalúa la participación.

* Para el logro de los objetivos más sencillos se trabaja de modo individual o en gran grupo, para objetivos más complejos se trabaja en pequeño grupo (de 3 a 5 miembros).

* No todos los miembros tienen porqué contribuir siempre por igual al grupo, aunque si es importante que interaccionen con los demás.

* Para las actividades más ambiguas y abiertas como el estudio de casos y la resolución de problemas se trabajará en pequeño grupo de 3 a 5 miembros, las actividades más predefinidas de modo individual y, en gran grupo, actividades como la lluvia de ideas.

6. Determinación de las posibilidades tecnológicas para apoyar el aprendizaje y la interacción.

* El trabajo se desarrollará a través de una página web a la que se accede mediante una clave personal.

* La comunicación e interacción se hará exclusivamente a través del ordenador: entre alumnos de diferentes institutos, el tiempo podrá ser sincrónico o asincrónico, es lo que Johansen (cfr. Fernández y otros, 1999) denomina "interacción distribuida sincrónica o asincrónica".

* Entre las funcionalidades para generar esta comunidad de aprendizaje en red, podemos enumerar:

a. Glosario: el glosario sobre los diferentes aspectos de las estrategias de autorregulación a ser trabajados se recogerán directamente aquí, en cambio los conceptos relacionados con el área académica (ciencias sociales, ciencias naturales...) sobre los que versen los textos serán incorporados por los propios alumnos a modo de trabajo en grupo.

b. Tablón: permite que el profesor presente las características del nuevo contenido de estudio así como las actividades, fechas y aclaraciones de 
diversa índole precisas.

c. Correo electrónico: para la comunicación asíncrona tanto con los compañeros como con el tutor.

d. Foro: para llevar a cabo actividades en gran grupo como sugerencias, aportaciones sobre una determinada cuestión, lluvia de ideas, etc.

e. Debate: espacio para el debate a nivel de gran grupo de cuestiones más problemáticas o ambiguas, presentación de las conclusiones, resultados y aportaciones del trabajo a nivel de pequeño grupo.

f. Chat: para la comunicación síncrónica con cualquier otro miembro de la comunidad.

g. Espacio de trabajo en pequeño grupo: con una zona de debate e intercambio para que el grupo desarrolle el trabajo y el profesor pueda observar la interacción e intervenir si lo considera oportuno. También contará con una zona de archivos a los que los alumnos van enviando sus aportaciones al trabajo común teniendo en cuenta el trabajo previo de sus compañeros.

h. Lista de preguntas más frecuentes: que se va engrosando (si el profesor lo considera pertinente) con nuevas dudas que van surgiendo y sus correspondientes respuestas.

\subsection{Características diferenciales de los roles.}

Las relaciones instruccionales establecidas a través de este entorno para el aprendizaje colaborativo se fundamenta en los diferentes niveles de comunicación e interacción en los que diversos elementos juegan un papel específico:

- Papel del profesor: iniciar a los alumnos, en un primer momento, en el manejo de la página web y sus diferentes apartados; monitorear la interacción de los grupos e intervenir cuando lo considere oportuno; diseñar actividades alternativas para el logro de los objetivos; evaluar el proceso de aprendizaje grupal e individual; realizar el feedback de las diferentes actividades e introducir las modificaciones oportunas; etc.

- Papel del alumno: interaccionar con los compañeros del grupo y con el material; cooperar en el desarrollo de las diferentes actividades propuestas; asumir progresivamente el control de su propio proceso de aprendizaje; ir tomando conciencia de su propio proceso de aprendizaje, de sus carencias y potencial de mejora, etc.

- Papel del grupo: facilitar el desarrollo y construcción del conocimiento mediante la confrontación de ideas, perspectivas e hipótesis de solución y abordaje de los problemas; generar una estructura de cooperación para lograr el éxito; disminuir el miedo al fracaso y al rechazo; aprovechar el potencial de todos los componentes del grupo y minimizar sus carencias; ayudar a los sujetos a centrase en el proceso para realizar la tarea al no propiciar la comparación social; fomentar la mutua aceptación, respeto y responsabilidad social; fomentar atribuciones de esfuerzo ante el éxito y el fracaso, etc.

- Papel de la tecnología: servir de facilitador y soporte de los procesos de comunicación anteriormente comentados, salvar las trabas en la comunicación e interacción por factores espacio-temporales.

4. Referencias bibliográficas.

BELTRÁN, J. (1998) Estrategias de aprendizaje, en SANTIUSTE, V. y BELTRÁN, J. (coords.) 
Dificultades de aprendizaje. Madrid, Síntesis, 201-237.

CASTELLS, M. (2001) Materiales para una teoría preliminar sobre la sociedad de redes, Revista de Educación, $\mathrm{n}^{\circ}$ extraordinario, 41-58.

COLL, C. (2004) Las Comunidades de Aprendizaje. IV Congreso Internacional de Psicología y Educación "Calidad Educativa", 1047-1060.

DURÁN, D. y MIQUEL, E. (2003) Cooperar para enseñar y aprender. Cuadernos de Pedagogía, 331, 73-76.

ECHEVERRÍA, J. (2002) ¿Internet en la escuela o la escuela en Internet?, Revista de Educación, ${ }^{\circ}$ extraordinario, 199-206.

ELBOJ, C. y OTROS (2002) Comunidades de aprendizaje. Transformar la educación. Barcelona, Graó.

FERNÁNDEZ, F. A. y OTROS (1999) Un entorno para aprendizaje colaborativo. Sistemas hipermedia y aula virtual, Revista de Enseñanza y Tecnología, Mayo-Agosto, 14-20.

GONZÁLEZ, M. C. (1999) La motivación académica. Sus determinantes y pautas de intervención. Navarra, EUNSA.

GROS, B. (2002) Constructivismo y diseños de entornos virtuales de aprendizaje, Revista de Educación, 328, 225-247.

JOCHEMS, W. M. G.; MARTENS, R. L. y STRIJBOS J. W. (2004) Designing for interaction: Six steps to designing computer-supported group-based learning, Computers \& Education, 42, 403-424.

NEO, M. (2003) Developing a collaborative learning environment using a web-based design, Journal of Computer Assisted Learning, 19, 462-473.

O.E.C.D. (2001) The PISA Project. Measuring students knowledge and skills for life. París, O.E.C.D.

(C) Ediciones Universidad de Salamanca 